\section{BMJ Open}

Ophthalmology

\title{
Strengthening retinopathy of prematurity screening and treatment services in Nigeria: a case study of activities, challenges and outcomes 2017-2020
}

Dupe S Ademola-Popoola (D) , ${ }^{1,2}$ Iretiola B Fajolu, ${ }^{3}$ Clare Gilbert, ${ }^{4}$ Bolutife A Olusanya, ${ }^{5}$ Oluwatoyin H Onakpoya, ${ }^{6}$ Chinyelu N Ezisi, ${ }^{7}$ Kareem O Musa, ${ }^{8}$ Robison Vernon Paul Chan, ${ }^{9}$ Valentina W Okeigbemen, ${ }^{10}$ Rilwan C Muhammad, ${ }^{11}$ Aeesha N J Malik, ${ }^{12}$ Adedayo O Adio, ${ }^{13}$ Olubunmi T Bodunde, ${ }^{14}$ Abdulkadir L Rafindadi, ${ }^{15}$ Tunji S Oluleye, ${ }^{16}$ Olukemi O Tongo, ${ }^{17}$ Sarat A Badmus, ${ }^{18}$ Olufunmilayo V Adebara, ${ }^{19}$ Tapas Ranjan Padhi (D) , ${ }^{20}$ Beatrice N Ezenwa, ${ }^{3}$ Tokunbo S Obajolowo, ${ }^{2}$ Lateefat B Olokoba, ${ }^{21}$ Victoria A Olatunji, ${ }^{21}$ Yewande Olubunmi Babalola, ${ }^{16}$ Mary O Ugalahi, ${ }^{5}$ Adetunji Adenekan, ${ }^{22}$ Omotayo O Adesiyun, ${ }^{23}$ Jagdish Sahoo, ${ }^{24}$ Marilyn T Miller, ${ }^{25}$ Odarosa M Uhumwangho, ${ }^{26}$ Adeduntan S Olagbenro, ${ }^{14}$ Ebunoluwa A Adejuyigbe, ${ }^{27}$ Chinyere V C Ezeaka, ${ }^{3}$ Olugbenga Mokuolu, ${ }^{28}$ Tinuade A Ogunlesi, ${ }^{29}$ Olusoga B Ogunfowora, ${ }^{29}$ Isa Abdulkadir, ${ }^{30}$ Fatima L Abdullahi, ${ }^{30}$ Abosede T Fabiyi, ${ }^{31}$ Laila $\mathrm{H}$ L Hassan, ${ }^{30}$ Aderonke M Baiyeroju, ${ }^{5}$ Peace I Opara, ${ }^{32}$ Kehinde Oladigbolu, ${ }^{15}$ Augusta U Eneh, ${ }^{32}$ Bassey E Fiebai, ${ }^{33}$ Fatima A Mahmud-Ajeigbe, ${ }^{15}$ Elijah N Peter, ${ }^{15}$ Hawwa S Abdullahi ${ }^{15}$

To cite: Ademola-Popoola DS, Fajolu IB, Gilbert C, et al. Strengthening retinopathy of prematurity screening and treatment services in Nigeria: a case study of activities, challenges and outcomes 2017-2020. BMJ Open Ophthalmology 2021;6:e000645. doi:10.1136/ bmjophth-2020-000645

Received 20 March 2021 Accepted 2 August 2021

\section{Check for updates}

(c) Author(s) (or their employer(s)) 2021. Re-use permitted under CC BY-NC. No commercial re-use. See rights and permissions. Published by BMJ.

For numbered affiliations see end of article.

Correspondence to Dr Dupe S Ademola-Popoola; dupsyp@yahoo.com

\section{ABSTRACT}

Objectives Retinopathy of prematurity (ROP) will become a major cause of blindness in Nigerian children unless screening and treatment services expand. This article aims to describe the collaborative activities undertaken to improve services for ROP between 2017 and 2020 as well as the outcome of these activities in Nigeria.

Design Descriptive case study.

Setting Neonatal intensive care units in Nigeria. Participants Staff providing services for ROP, and 723 preterm infants screened for ROP who fulfilled screening criteria (gestational age $<34$ weeks or birth weight $\leq 2000 \mathrm{~g}$, or sickness criteria).

Methods and analysis A WhatsApp group was initiated for Nigerian ophthalmologists and neonatologists in 2018. Members participated in a range of capacitybuilding, national and international collaborative activities between 2017 and 2018. A national protocol for ROP was developed for Nigeria and adopted in 2018; 1 year screening outcome data were collected and analysed. In 2019 , an esurvey was used to collect service data from WhatsApp group members for 2017-2018 and to assess challenges in service provision.

Results In 2017 only six of the 84 public neonatal units in Nigeria provided ROP services; this number had increased to 20 by 2018 . Of the 723 babies screened in 10 units over a year, 127 (17.6\%) developed any ROP; and 29 (22.8\%) developed type 1 ROP. Only 13 (44.8\%) babies were treated, most by intravitreal bevacizumab. The screening criteria were revised in 2020 . Challenges included lack of equipment to regulate oxygen and to document and treat ROP, and lack of data systems. Conclusion ROP screening coverage and quality improved after national and international collaborative efforts. To scale up and improve services, equipment for neonatal care and ROP treatment is urgently needed, as well as systems to monitor data. Ongoing advocacy is also essential.

\section{INTRODUCTION}

Africa and Asia are responsible for over half $(52 \%)$ of live births worldwide and over $80 \%$ of babies born preterm. Worldwide, 9 of the 11 countries with preterm birth rates over $15 \%$ are in sub-Saharan Africa (SSA). ${ }^{12}$ Nigeria has about 7141000 births annually, approximately 803000 of which are born at less than 37 weeks gestational age (GA), the third highest after India and China. About 43800 of these babies have a GA of less than 28 weeks. $^{2}$

Over the last decade, there have been notable improvements in human and technical resource capacity for health in SSA. In particular, the number of trained ophthalmologists available to perform retinopathy of prematurity (ROP) screening has recently 


\section{Key messages}

\section{What is already known about this subject?}

Retinopathy of prematurity (ROP), a vasoproliferative disease of the retina that occurs in preterm and low birth weight babies, is becoming more common in Nigeria as a result of better neonatal survival rates. Screening and prompt treatment, although technology-dependent, are vital for preventing blindness from ROP. There are regional variations in screening criteria based on levels of socioeconomic development.

\section{What are the new findings?}

- Leadership, advocacy, networking and collaborative teamwork were important for the initiation and enhancement of ROP services. Development of a national screening protocol facilitated the detection, treatment and reporting of sight-threatening ROP in Nigerian babies. Major challenges encountered included inadequacy of equipment for monitoring oxygen delivery, lack of wide field imaging systems for documentation, and lack of laser equipment.

\section{How might these results change the focus of research or} clinical practice?

- The findings of this study have resulted in a modification of national screening criteria and also provide further evidence to strengthen on-going advocacy to both governmental agencies and nongovernmental organizations for the provision of equipment and facilities for ROP services.
With regards to screening criteria, 'one size does not fit all' as larger, more mature infants also develop type 1 ROP in resource-limited settings than in well resourced settings. ${ }^{12}$ Variation in the population of preterm infants at risk is reflected in national ROP screening guidelines in many low and middle income countries, including South Africa $(2013){ }^{13}$ and Kenya (2018) ${ }^{14}$ In these countries the screening criteria include a GA of up to 32 weeks, or a BW of up to $1501 \mathrm{~g}$; or infants with a GA greater than 32-35 weeks or BW of 1501-2000 g with comorbidities, at the request of the neonatologist. ${ }^{13}{ }^{14}$ In India the criteria are wider; that is, GA 4 weeks or BW of up to $2000 \mathrm{~g} .{ }^{15}$

In Nigeria, the most populous country in Africa, there are 42 federal government tertiary hospitals with large NICUs ${ }^{16}$ and a similar number of state government hospitals also provide neonatal services. In 2016, there was no national protocol for ROP screening. At that time, the six hospitals providing ROP services used different criteria, with GA criteria ranging from less than 32 weeks to less than 35 weeks, and BW criteria from less than $1200 \mathrm{~g}$ to less than $1900 \mathrm{~g} .{ }^{17-19}$

This case study describes the processes and collaborative activities undertaken to promote ROP services in NICUs in Nigeria following the realisation that ROP is an emerging public health problem. The outcomes of these efforts are also reported.

\section{METHODS}

This was a descriptive case study. After the perceived increase in blindness from ROP observed in late 2016, a number of activities and processes were initiated (figure 1). This included advocacy for screening, enhancement of communication and collaboration between professional colleagues, capacity building and skills transfer, and the development of a national ROP screening protocol. In addition, an esurvey was undertaken to assess the status of essential equipment for neonatal care and ROP, and the challenges encountered in delivering services in Nigeria. In addition, data from the first collaborative ROP screening activity in Nigeria using the new protocol were collected and analysed.

\section{Advocacy for ROP screening}

Advocacy efforts were started in 2017 by local leaders in ROP care by engaging members of the Nigerian Paediatric Ophthalmology and Strabismus Society (NIPOSS). Efforts were directed towards critical stakeholders, including hospital administrators, neonatologists and paediatricians, NICU nurses, ophthalmologists, midwives and obstetricians, parents of preterm infants, and the general public through short radio jingles. In addition, presentations on ROP and its blinding consequences on the child, their family and caregivers, including medico-legal implications, were delivered at three national society meetings of ophthalmologists, paediatricians and neonatologists.

\section{Communication and collaboration}

A social media (WhatsApp) chat group was formed, the 'Nigeria ROP Team', to improve communication, 


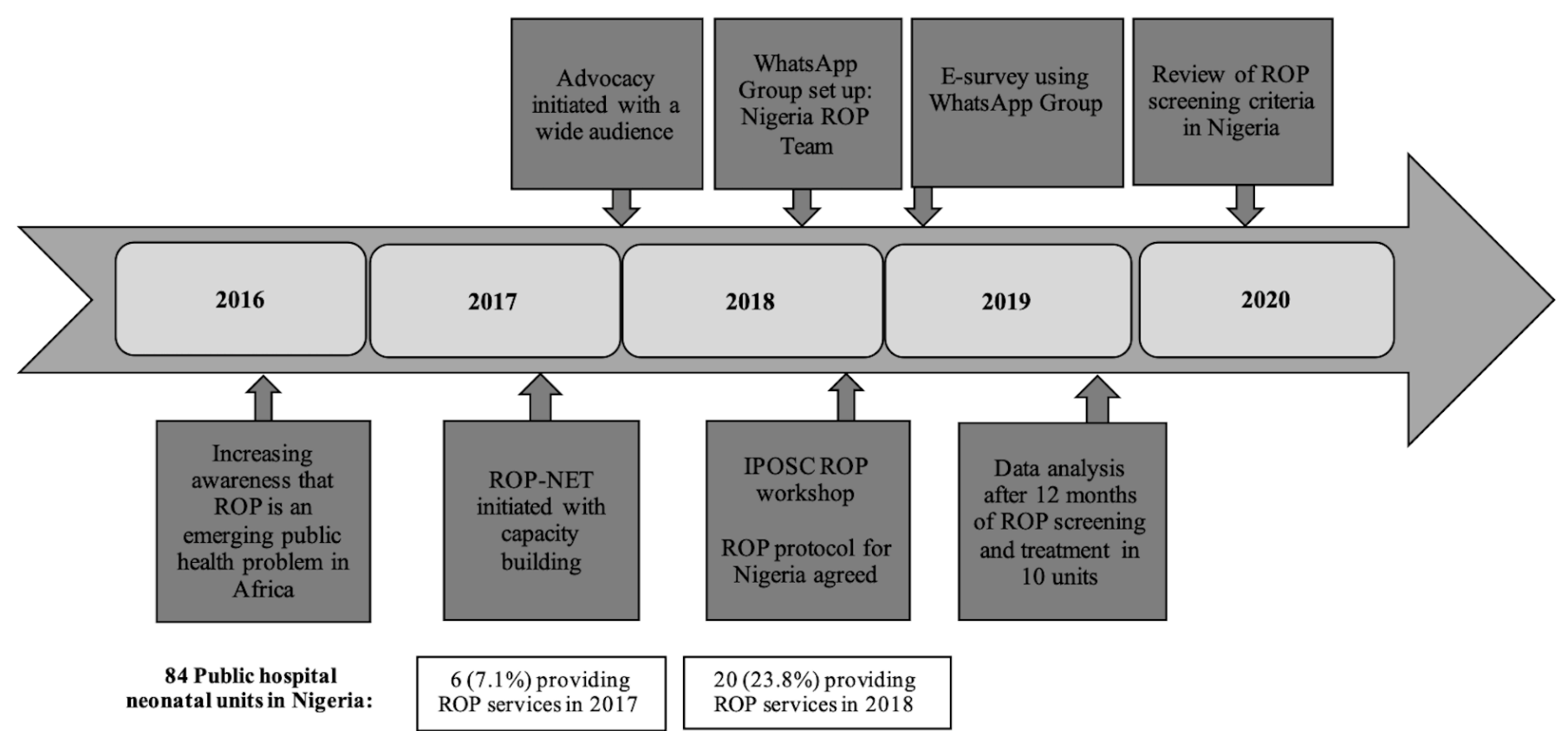

Figure 1 Activities for retinopathy of prematurity (ROP) in Nigeria 2016-2020. IPOSC, International Pediatric Ophthalmology and Strabismus Council.

collaboration, and to share experiences and resources among ROP teams on 8 August 2018. Links to join the groups were shared with members of NIPOSS, the VitreoRetinal Society of Nigeria (VRSN) and the Nigeria Society for Neonatal Medicine (NISONM).

\section{Capacity building and skills transfer}

Capacity-building opportunities were provided by the UK's Queen Elizabeth Diamond Jubilee Trust (The Trust; 2012-2019) through the Commonwealth Eye Health Consortium's fellowship programme, which included a south-south Retinopathy of Prematurity Network (ROP-NET).$^{20}$ Between 2014 and 2019, 18 Nigerian ophthalmologists underwent paediatric ophthalmology training fellowships in designated training institutions, several of which were in India where ROP services are expanding. The ROP-NET supported capacity building, quality improvement and scale-up of ROP screening through a partnership between the University of Ilorin Teaching Hospital, Nigeria and centres of excellence in India between 2017 and 2019. The ophthalmology partnership was with ROP experts in LV Prasad Eye Institute, Bhubaneswar, India and with neonatal partners from the Institute of Medical Sciences and Sum Hospital in India. Activities included joint planning meetings followed by reciprocal visits for workshops and hands-on training in ROP screening and treatment.

\section{Development of ROP screening protocol}

The International Paediatric Ophthalmology and Strabismus Council (IPOSC) ran a workshop on ROP for African countries in Cape Town in September 2018. The Nigerian participants, including neonatologists, ophthalmologists and NICU nurses from five institutions, held a face-to-face meeting chaired by one of the authors (DAP), a paediatric ophthalmologist and Chair of NIPOSS at the time, and reached a consensus on the need for a standardised, national protocol for ROP for Nigeria. Interim screening criteria and timing of the first screening were agreed by consensus, and it was agreed that evidence on the population at risk of type $1 \mathrm{ROP}$ was needed to refine the criteria.

The protocol, which was finalised within a month of the meeting by email, was shared with participants from 35 Nigerian institutions on the WhatsApp group. The agreed criteria for screening were: (1) infants with GA of up to 34 weeks or BW of less than $2000 \mathrm{~g}$; (2) late preterm infants ( $>34$ but $<37$ weeks) if exposed to risk factors (prolonged oxygen supplementation, multiple episodes of apnoea, blood transfusion, respiratory distress syndrome, intraventricular haemorrhage or sepsis); or at the request of NICU staff because of stormy postnatal events. The first screening should occur around 28 days of life, or earlier if the infant was to be discharged before 28 days. Screening before discharge in these settings has been shown to increase follow-up after discharge. ${ }^{21}$ This initial protocol was presented to relevant authorities in the Federal Ministry of Health and was adopted for use in the development of the treatment guidelines for child eye health services in Nigeria. ${ }^{22}$ Patient records were paper based, and data for this study were extracted and entered into a Microsoft Excel spreadsheet. The characteristics of the babies who developed type 1 ROP were used to review the screening protocol at a virtual meeting in July 2020.

\section{E-survey}

In order to assess the status of ROP services in Nigeria, an online survey was used to collect information separately 
for the years 2017 and 2018 on the duration, frequency and regularity of ROP screening, participants' confidence in providing services for ROP, and challenges they had encountered in screening and treatment. The questionnaire was designed by one of the authors (DAP) and sent to a few key members (ophthalmologists and neonatologists) of the WhatsApp group for feedback and comments. The final questionnaire was created in Google forms, where the first question was to obtain consent to participate. The survey, which had 10 closed questions and one free text question on challenges encountered with ROP services, was not advertised and did not include consistency checks. All questions were compulsory and particpants were not able to change their initial responses. All items provided a non-response option such as 'not applicable' in line with CHERRIES checklist recommendations. ${ }^{23} \mathrm{~A}$ link to the form was sent to all members of the Nigeria ROP Teams WhatsApp group. In the invitation, members were requested to designate one individual (an ophthalmologist or neonatologist) per institution to complete the form. The email address of this individual was used to $\log$ onto the esurvey, which allowed participants' institutions to be identified as well as duplicate records. The link was sent on 1 January 2019 and responses were requested by the end of the month. Data were then exported into Excel, and extracted and analysed for this paper using frequency distributions.

Patients and the public were not involved in the design, conduct, reporting, or dissemination plans of this study.

\section{RESULTS}

Outcome of advocacy, communication, capacity building and skills transfer

By August 2019 the 'ROP Team Nigeria' WhatsApp group had 40 participants: 18 ophthalmologists, 12 neonatologists/paediatricians and 10 NICU nurses.

\section{National and international collaboration meetings for capacity} building and skills transfer

The number of tertiary level public NICUs in Nigeria was 84 ; in 2017 , six were providing ROP services which increased to 20 in 2018. Screening was weekly and regular in $15(75 \%)$ NICUs, but was but irregular or infrequent in five. Three ROP-blind children were seen in 2017 and

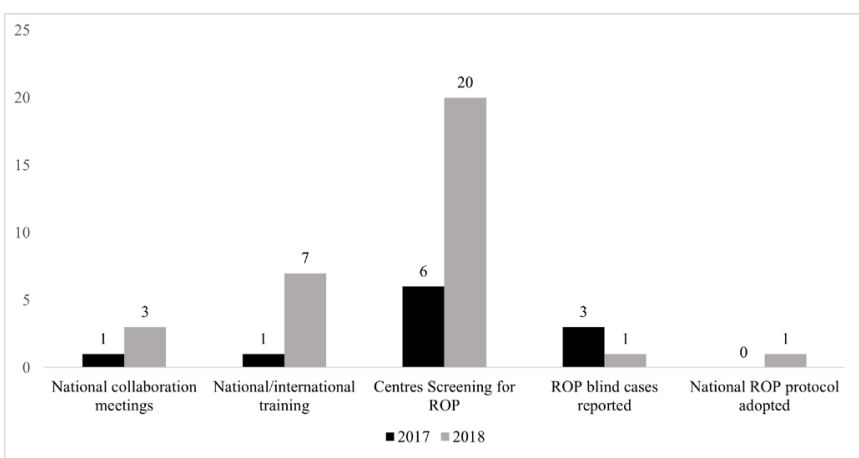

Figure 2 Status of retinopathy of prematurity (ROP) programme in Nigeria in 2017 and 2018. one in 2018 (figure 2). The number of ophthalmologists confident in ROP screening increased from 10 to 23 over the same period.

\section{ROP screening and treatment}

Following development of the protocol, 10 ROP teams in tertiary hospitals across Nigeria participated in a 1 year prospective study of ROP using the protocol, from September 2018 to August 2019. A total of 723 infants were screened, $475(65.7 \%)$ had GA of less than 32 weeks and $71(9.8 \%)$ had a GA of more than 32 weeks, while $517(71.5 \%)$ had a BW of up to $1500 \mathrm{~g}$. A total of 127 $(17.6 \%)$ babies developed any ROP; $29(4.0 \%)$ had type 1 ROP. The median GA of infants with type 1 ROP was 28 weeks (IQR 27-29 weeks), and the median BW was 1034.3 g (IQR 935-1174 g); a comparatively large number of babies were examined to diagnose ROP in babies with birth weight over $1200 \mathrm{~g}$ and beyond 31 weeks (table 1 , figure 3).

Only one infant developed aggressive posterior ROP (APROP). Twenty-seven of the 29 babies with type 1 ROP had a GA of less than 32 weeks $(27 / 475,5.2 \%)$ or a BW of $1500 \mathrm{~g}(27 / 517,5.6 \%)$. The two infants with type 1 ROP with a GA of at least 32 weeks and a BW of more than $1500 \mathrm{~g}$ had experienced a stormy neonatal period, with Candida sepsis and multiple apnoeic attacks. Other risk factors for ROP included anaemia in 19 (65.5\%), apnoea/respiratory distress syndrome and sepsis, each in $18(62.1 \%)$ babies.

Only $13(44.8 \%)$ of the 29 infants with type 1 ROP were treated: one had primary diode 810 laser treatment, and $12(92.3 \%)$ had anti-vascular endothelial growth factor (VEGF; bevacizumab) injections because this was the only treatment available in $90 \%$ of the institutions. Two $(16.7 \%)$ of these 12 infants subsequently had laser treatment for persistent disease. In 12 infants, the ROP regressed; in one, it progressed to stage $4 \mathrm{~b}$. Sixteen infants $(56.2 \%)$ were not treated; 14 parents could not afford the treatment, one did not consent, and one infant died within 24 hours of the diagnosis.

At a meeting on 21 July 2020, the Nigerian ROP Team took a unanimous decision to adjust the $\mathrm{BW}$ component of the ROP screening criteria, from up to $2000 \mathrm{~g}$ to up to $1500 \mathrm{~g}$, while maintaining the GA criterion of up to 34 weeks. Neonatologists may still request screening for more mature or heavier infants if they have been exposed to risk factors. It was agreed that these criteria would be used for another year, after which they would be reviewed again.

\section{Online E-survey findings}

Twenty Nigerian institutions offering ROP services responded to the online e-survey. Five were not providing regular services, and the challenges encountered in the 15 institutions providing regular services are reported (table 2). The main challenge in all the institutions was a lack of wide-field imaging systems to document findings even though smartphones were used for retinal imaging 
Table 1 Gestational age and birth weight of all babies screened and the 29 with type 1 ROP in Nigeria

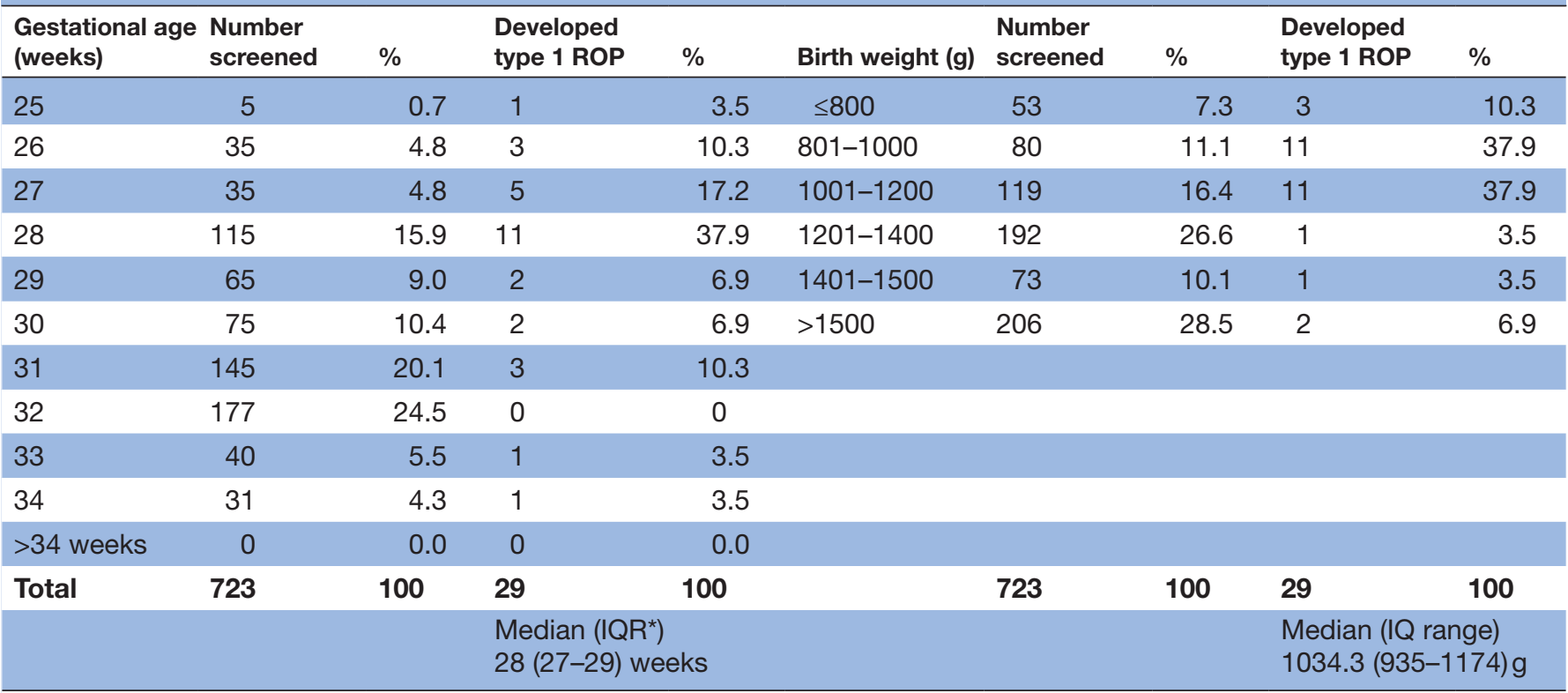

${ }^{*} \mathrm{IQR}$.

ROP, retinopathy of prematurity .

by two institutions. Lack of equipment to regulate oxygen in NICUs was another universal challenge. Two-thirds of institutions lacked a laser for ROP treatment.

\section{DISCUSSION}

ROP emerged as an increasingly important cause of blindness in children in middle-income countries over 20 years ago. ${ }^{24}$ More recently this 'third epidemic of ROP blindness' emerged in South Africa ${ }^{25}$ and in other lowincome African countries. ${ }^{26} 27$ Few studies on ROP were published from SSA before the year $2000,{ }^{19} 28$ which probably reflects the very high mortality of preterm infants at the time.

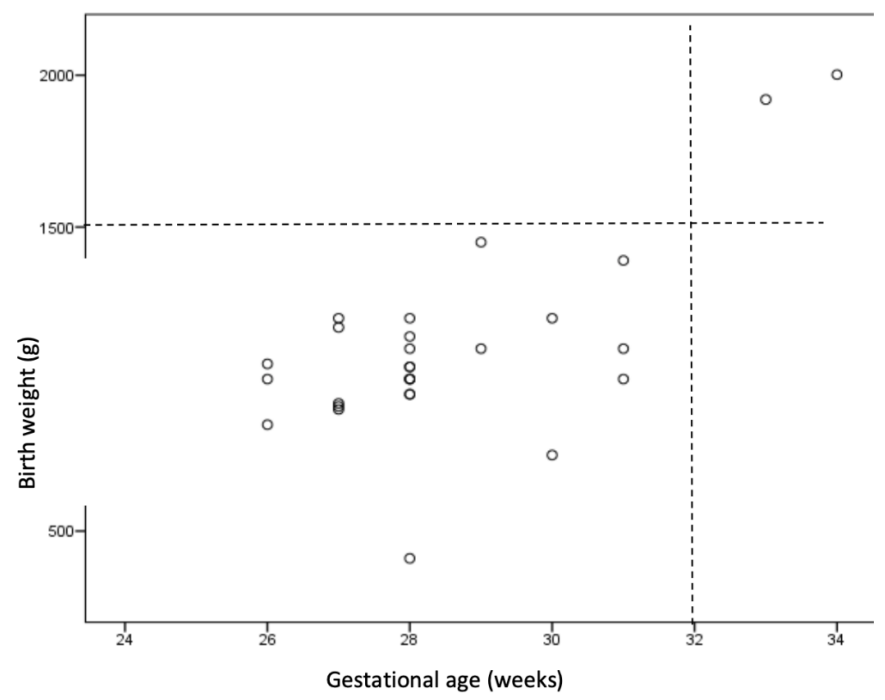

Figure 3 Birth weight and gestational ages of babies developing type 1 retinopathy of prematurity (ROP).
In 2017, only six of the 84 NICUs in government-owned institutions in Nigeria provided regular ROP screening, which had increased to 20 by early 2019. More work needs to be done to increase the number of centres screening and managing ROP. In terms of human resources, the number of ophthalmologists with relevant competencies for ROP screening and management increased

Table 2 Challenges in the 15 institutions delivering routine ROP screening in Nigeria

\begin{tabular}{|c|c|c|}
\hline Challenges & Frequency & $\%$ \\
\hline \multicolumn{3}{|l|}{ Lack of equipment and consumables } \\
\hline $\begin{array}{l}\text { Electronic systems to document } \\
\text { ROP findings }\end{array}$ & 15 & 100 \\
\hline $\begin{array}{l}\text { Lack of equipment to regulate } \\
\text { oxygen in NICUs }\end{array}$ & 15 & 100 \\
\hline No laser for treatment & 10 & 66.7 \\
\hline $\begin{array}{l}\text { Other: indirect ophthalmoscopes, } \\
\text { specula, eye drops }\end{array}$ & 4 & 26.7 \\
\hline \multicolumn{3}{|l|}{ Other challenges } \\
\hline Data management & 13 & 86.7 \\
\hline Financial support for treatment & 10 & 66.7 \\
\hline Late referral & 6 & 40.0 \\
\hline $\begin{array}{l}\text { ROP coordinator/support staff/ } \\
\text { inadequate manpower }\end{array}$ & 4 & 26.7 \\
\hline $\begin{array}{l}\text { Poor motivation and transportation } \\
\text { issues for parents and team }\end{array}$ & 4 & 26.7 \\
\hline Follow-up & 4 & 26.7 \\
\hline
\end{tabular}

NICU, neonatal intensive care unit; ROP, retinopathy of prematurity. 
from 10 to 23 over the same period, largely due to the capacity-building opportunities provided by the Trust's south-south ROP-NET partnership and the CEHC fellowship programme.

Collaborative efforts between professional societies of ophthalmologists and neonatologists in Nigeria as well as other non-governmental organisations resulted in the development of the first protocol for ROP. This not only standardised the provision of services, but as has been shown in South American countries, the development of guidelines is a mechanism for engaging ministries of health, which is key to scaling up services. ${ }^{29} 30$

The outcome of these activities provides further evidence to support the positive impact of leadership, collaboration and advocacy in developing, implementing and scaling up services for greater coverage and alignment of services. ${ }^{29}$ This was demonstrated by the more than three-fold increase in the number of institutions providing ROP services in Nigeria over a 3-year period.

In our study, $4.0 \%$ of the babies screened in 10 NICUs developed type 1 ROP, comparable to reports from other SSA countries (1-8.7\%). 25263132 The proportion of infants with any ROP was lower than reported in other studies from Africa, which reflect narrower screening criteria used in some of these countries. Most of the infants with type 1 ROP had a GA of less than 32 weeks or a BW of less than $1500 \mathrm{~g}$, and there were two outliers, one with a GA of 34 weeks. These findings supported the Nigerian team's decision to keep the GA screening criteria at up to 34 weeks GA and adjust the BW to $1500 \mathrm{~g}$ or lower, and the inclusion of older or bigger infants with stormy clinical course. The finding that approximately $5 \%$ of infants with a GA less than 32 weeks or a BW up to $1500 \mathrm{~g}$ developed type 1 ROP despite the relatively high median GA (28 weeks) suggests that more needs to be done to improve the quality of neonatal care in Nigeria.

In this study, less than half of the infants with Type 1 ROP were treated, either due to lack of lasers or parental consent, or the high cost of anti-VEGF treatment or infant death. This situation needs to be addressed as a matter of urgency through advocacy, mobilisation of resources, better counselling of parents, and by ensuring that ROP management is included in Universal Health Coverage financing schemes.

The use of anti-VEGF agents in the management of ROP is becoming increasingly popular globally, ${ }^{33-35}$ including in this study where, in the absence of lasers, it was the primary treatment in most infants (92.3\%). Advantages of anti-VEGF treatment over laser include that it is quicker to administer, can give early resolution of active disease, and may be associated with fewer cases of early-onset high myopia. However, the disadvantages are late reactivation of disease, as occurred in two infants in this study, which can occur many months later in an unpredictable manner. Most importantly, there is potential for systemic complications due to the suppression of endogenous VEGF while many organs are still developing. Determining which is the best anti-VEGF agent for ROP, and the indications, timing and dose required, are active areas of research. ${ }^{36}$ For example, a recent dosing study suggests that $0.004 \mathrm{mg}$ bevacizumab may be the lowest effective dose $^{37}$; that is, $1 / 150$ th of the $0.625 \mathrm{mg}$ dose used in the BEAT-ROP trial, but evidence from clinical trials is needed before doses can be reduced to these levels.

While most centres conducted regular weekly screening, five reported infrequent screening (some were screening at quarterly intervals). This is concerning as babies with type 1 ROP are likely to be missed. Efforts to increase neonatologists' ownership and awareness are required. Systems to collect and monitor data on ROP services, preferably electronic, are also required so that data can be compared between units and tracked over time, and to identify the uptake and completeness of screening and the outcome of treatment.

Also highlighted is the need for peer support for training in screening and treatment with provision of equipment. A lack of skilled ophthalmologists is likely to remain a considerable constraint to scaling up services in the future, particularly for screening using indirect ophthalmoscopy. Under these circumstances, telemedicine as used in India ${ }^{38}$ has much to offer; retinal images are captured by highly competent, visiting teams of trained technicians, with remote image analysis by an ROP expert. Real-time image analysis using artificial intelligence to identify infants with plus disease, a key sign of type 1 ROP, is also on the horizon, ${ }^{39}$ with great potential for scaling up services.

As in many low-income and middle-income countries, there were challenges with delivering high-quality neonatal care to ensure that sick newborns survive and thrive. ${ }^{40}$ Exposure to hyperoxia and fluctuating hypoxiahyperoxia are well recognised risk factors for ROP. In the 15 Nigerian institutions included in this study, there was a complete lack of air-oxygen blenders and an inadequate number of pulse oximeters to monitor oxygen saturation. This may explain the relatively high proportion of infants with type 1 ROP despite the relatively broad screening criteria. Advocacy with the Ministry of Health is required to ensure that policies for sick and preterm newborns include the safe delivery of oxygen from immediately after birth, with target saturations, as recommended by WHO. ${ }^{4}$ Ensuring these policies are in place and are implemented is critical as neonatal care services continue to expand.

\section{CONCLUSIONS}

ROP services in Nigeria have improved over the past 3 years as a result of local leadership and several national and international collaborative efforts. More effort will be required to scale up services to other NICUs using the revised screening criteria. In particular, there is an urgent need to provide wide-field imaging systems for screening which can be done by non-ophthalmologists, and lasers and anti-VEGF for treatment, with financing mechanisms which ensure that no infants are denied treatment because parents cannot afford it. To prevent ROP, advocacy with the Ministry of Health is required to 
establish national protocols for safe oxygen delivery in all NICUs. Continuous collaboration and ongoing advocacy for support from all stakeholders, government, international and local NGOs are required to control blindness from ROP in Nigeria.

Further research is required using the revised criteria; this might help reduce the high number needed to diagnose ROP in babies with birth weight over $1200 \mathrm{~g}$ and beyond 31 weeks, thereby improving efficiency of ROP programmes.

\section{Author affiliations}

${ }^{1}$ Department of Ophthalmology, University of Ilorin, Ilorin, Kwara, Nigeria

${ }^{2}$ Paediatric Ophthalmology and Strabismus Unit, Department of Ophthalmology, University of Ilorin Teaching Hospital, Ilorin, Kwara, Nigeria

${ }^{3}$ Department of Neonatology/Perinatology, Paediatrics, University of Lagos College of Medicine/ Lagos University Teaching Hospital, Lagos, Nigeria

${ }^{4}$ Department of Clinical Research, London School of Hygiene and Tropical Medicine, London, UK

${ }^{5}$ Paediatric Ophthalmology \& Strabismus Unit, Department of Ophthalmology, College of Medicine, University of Ibadan and University College Hospital, Ibadan, Oyo, Nigeria

${ }^{6}$ Retinal Unit, Department of Ophthalmology, Obafemi Awolowo University Teaching Hospital, Ile-Ife, Osun, Nigeria

${ }^{7}$ Pediatric Ophthalmology and Strabismus Unit, Department of Ophthalmology, Alex Ekwueme Federal University Teaching Hospital, Abakaliki, Ebonyi, Nigeria ${ }^{8}$ Paediatric Ophthalmology and Strabismus Unit, Department of Ophthalmology, Lagos University Teaching Hospital, Idi-araba, Lagos, Nigeria

${ }^{9}$ Department of Ophthalmology and Visual Sciences, Illinois Eye and Ear Infirmary, University of Illinois at Chicago, Chicago, Illinois, USA

${ }^{10}$ Paediatric Ophthalmology Unit, Department of Ophthalmology, University of Benin Teaching Hospital, Benin City, Edo, Nigeria

${ }^{11}$ Department of Ophthalmology, University of Abuja Teaching Hospital,

Gwagwalada, Abuja, Nigeria

${ }^{12}$ London School of Hygiene and Tropical Medicine, London, UK

${ }^{13}$ Paediatric Ophthalmology Unit, Department of Ophthalmology, University of Port Harcourt Teaching Hospital, Port Harcourt, Rivers, Nigeria

${ }^{14}$ Department of Ophthalmology, Olabisi Onabanjo University Teaching Hospital, Sagamu, Ogun State, Nigeria

${ }^{15}$ Department of Ophthalmology, Ahmadu Bello University Teaching Hospital, Zaria, Kaduna, Nigeria

${ }^{16}$ Vitreoretinal Unit, Department of Ophthalmology, College of Medicine, University of Ibadan and University College Hospital, Ibadan, Oyo, Nigeria

${ }^{17}$ Department of Paediatrics, College of Medicine, University of Ibadan and University College Hospital, Ibadan, Oyo, Nigeria

${ }^{18}$ Paediatric Ophthalmology Unit, Department of Ophthalmology, Obafemi Awolowo University Teaching Hospital, lle-lfe, Osun State, Nigeria

${ }^{19}$ Neonatal Unit, Department of Paediatrics, University of llorin Teaching Hospital, Ilorin, Kwara, Nigeria

${ }^{20}$ Vitreoretinal Services, LV Prasad Eye Institute Bhubaneswar Campus,

Bhubaneswar, India

${ }^{21}$ Vitreoretinal Unit, Department of Ophthalmology, University of Ilorin Teaching

Hospital, llorin, Kwara, Nigeria

${ }^{22}$ Vitreoretinal Unit, Department of Ophthalmology, University of Lagos, Lagos, Nigeria

${ }^{23}$ Department of Paediatrics, University of Ilorin Teaching Hospital, Ilorin, Kwara, Nigeria

${ }^{24}$ Neonatology, IMS and SUM Hospital, Kalinga Nagar, Bhubaneswar, Odisha, India, Bhubaneswar, Odisha, India

${ }^{25}$ Department of Ophthalmology, University of Illinois College of Medicine, Chicago, Illinois, USA

${ }^{26}$ Vitreoretinal Unit, Department of Ophthalmology, University of Benin Teaching Hospital, Benin City, Edo, Nigeria

${ }^{27}$ Peadiatrics and Child Health, Obafemi Awolowo University Teaching Hospital Complex, lle-lfe, Osun State, Nigeria

${ }^{28}$ Neonatal Unit, Department of Paediatrics, University of Ilorin, Ilorin, Kwara, Nigeria
${ }^{29}$ Department of Paediatrics, Olabisi Onabanjo University Teaching Hospital, Sagamu, Ogun State, Nigeria

${ }^{30}$ Department of Paediatrics, Ahmadu Bello University Teaching Hospital, Zaria, Kaduna, Nigeria

${ }^{31}$ Special Care Baby Unit, Nursing Service, Ahmadu Bello University Teaching Hospital, Zaria, Kaduna, Nigeria

${ }^{32}$ Department of Paediatrics, University of Port Harcourt Teaching Hospital, Port Harcourt, Rivers, Nigeria

${ }^{33}$ Vitreoretinal Unit, Department of Ophthalmology, University of Port Harcourt Teaching Hospital, Port Harcourt, Rivers, Nigeria

Acknowledgements International Paediatric Ophthalmology and Strabismus Council-IPOSC; UK's Queen Elizabeth Diamond Jubilee Trust's Commonwealth Eye Consortium which included fellowship training and support to the ROP-NET, Federal Ministry of Health-FMOH; Nigeria Paediatric Ophthalmology and Strabismus Society-NIPOSS; Vitreoretinal Society of Nigeria-VRSN and the Nigeria Society for Neonatal Medicine-NISONM. NICU staff and caregivers are also acknowledged.

Contributors The authors made substantial contributions to the conception or design of the work; or the acquisition, analysis, or interpretation of data for the work; drafting the work or revising it critically for important intellectual content final approval of the version to be published; and agreement to be accountable for all aspects of the work in ensuring that questions related to the accuracy or integrity of any part of the work are appropriately investigated and resolved.

Funding Some of the activities were supported by the UK's Queen Elizabeth Diamond Jubilee Trust's Commonwealth Eye Consortium which included fellowship training and support to the ROP-NET and International Paediatric Ophthalmology and Strabismus Society, which supported the ROP workshop in Cape Town, South Africa.

Competing interests None declared.

Patient and public involvement Patients and/or the public were not involved in the design, or conduct, or reporting, or dissemination plans of this research.

Patient consent for publication Not required.

Ethics approval The study was conducted in accordance with the ethical standards of the National Health Research Ethics Committee of Nigeria-NHREC and with the Declaration of Helsinki. NHREC Approval Number NHREC/01/01/2007-04/12/2020. ABUTHZ/HREC/w34/2019

Provenance and peer review Not commissioned; externally peer reviewed.

Data availability statement Data are available upon reasonable request.

Open access This is an open access article distributed in accordance with the Creative Commons Attribution Non Commercial (CC BY-NC 4.0) license, which permits others to distribute, remix, adapt, build upon this work non-commercially, and license their derivative works on different terms, provided the original work is properly cited, appropriate credit is given, any changes made indicated, and the use is non-commercial. See: http://creativecommons.org/licenses/by-nc/4.0/.

ORCID iDs

Dupe S Ademola-Popoola http://orcid.org/0000-0002-3867-1948

Tapas Ranjan Padhi http://orcid.org/0000-0002-7580-3225

\section{REFERENCES}

1 March of Dimes. Save the Children, WHO. In: Howson CP, Kinney MV, Lawn JE, eds. Born too soon: the global action report on preterm birth born too soon. World Health Organization, 2012.

2 Nigeria profile of preterm and low birth weight prevention and care. Available: https://www.everypreemie.org/wp-content/uploads/2019/ 07/Nigeria_7.5.19.pdf [Accessed 17th Apr 2020].

3 Lee LA. Commonwealth eye health Consortium final evaluation, 2019. Available: https://www.iapb.org/wp-content/uploads/TrustCommonwealth-Eye-Health-Consortium-Final-Evaluation.pdf [Accessed 22nd May, 2020].

4 World Health Organisation. Oxygen therapy for children: a manual for health workers, 2017. Available: http://www.who.int/maternal child adolescent/documents/child-oxygen-therapy/en/ [Accessed 24 Jun 2020].

5 WHO. Born too soon: the global action report on preterm birth, 2016.

6 Bako B, Idrisa A, Garba MA, et al. Determinants of neonatal survival following preterm delivery at the University of Maiduguri teaching Hospital, Maiduguri, Nigeria. Trop J Obstet Gynaecol 2017;34:39. 
7 Neonatal Intensive Care Unit, UITH I. Nicu 2019 annual morbidity and mortality review, 2019 Ilorin annual paediatric digest, 2019.

8 NICU Neonatal Intensive Care Unit, University of Ilorin Teaching Hospital N. Ilorin paediatric digest. Nigeria: Department of Paediatrics, University of Ilorin teaching Hospital, 2019.

9 Ademola-Popoola DS, Oluleye TS. Retinopathy of prematurity (ROP) in a developing economy with improving health care. Curr Ophthalmol Rep 2017;5:114-8.

10 Asferaw M, Gilbert C. Alemu Kerie Retinopathy of prematurity - an emerging cause of childhood blindness in Ethiopia. Ethiop Med J 2020;58 https://www.emjema.org/index.php/EMJ/article/view/1377

11 Gilbert C, Malik ANJ, Nahar N, et al. Epidemiology of ROP update Africa is the new frontier. Semin Perinatol 2019;43:317-22.

12 Gilbert CE. Screening for retinopathy of prematurity: does one size fit all? Arch Dis Child Fetal Neonatal Ed 2016;101:F280-1.

13 Sitati S, Mwangi N, Njambi L. National guidelines for screening and management of retinopathy of prematurity in Kenya: an overview of the recommendations. J Ophthalmol Eastern Central Southern Afr 2019;23:3-5

14 Visser L, Singh R, Young M. Guideline for the prevention, screening and treatment of retinopathy of prematurity (ROP). South African Med J 2013;103 https://hdl.handle.net/10520/EJC130733

15 Shukla R, Murthy GVS, Gilbert C, et al. Operational guidelines for ROP in India: a summary. Indian J Ophthalmol 2020;68:108-14.

16 Federal Ministry of Health Nigeria. List of teaching hospitals in Nigeria. Available: http://health.gov.ng/index.php?option=com content\&view $=$ article\&id=136\&ltemid=502 [Accessed 22 May 2020].

17 Ademola-Popoola D, Adesiyun O, Durotoye IA, et al. Screening programme for retinopathy of prematurity in Ilorin, Nigeria: a pilot study. West Afr J Med 2013;32:281-5.

18 Fajolu IB, Rotimi-Samuel A, Aribaba OT, et al. Retinopathy of prematurity and associated factors in Lagos, Nigeria. Paediatr Int Child Health 2015;35:324-8.

19 Adio AO, Ugwu RO, Nwokocha CG, et al. Retinopathy of prematurity in Port harcourt, Nigeria. ISRN Ophthalmol 2014;2014:1-6.

20 Malik A. The retinopathy of prematurity network (ROP-NET): bringing essential eye care to premature babies, 2018. Available: https:// www.iapb.org/news/the-retinopathy-of-prematurity-network-ropnet-bringing-essential-eye-care-to-premature-babies/

21 Vinekar A, Jayadev C, Mangalesh S, et al. Initiating retinopathy of prematurity screening before discharge from the neonatal care unit: effect on Enrolment in rural India. Indian Pediatr 2016;53 Suppl 2:S107-11.

22 Federal Ministry of Health. Retinopathy of prematurity. in: treatment guidelines for delivery of child eye health services in Nigeria. Abuja, 2019. Available: https://www.health.gov.ng/doc/TreatmentGuidelines-for-Delivery-of-Child-Eye-Health\%20Services\%20in\% 20Nigeria.pdf

23 Eysenbach G. Improving the quality of web surveys: the checklist for reporting results of Internet E-Surveys (cherries). J Med Internet Res 2004;6:e34.

24 Gilbert C, Rahi J, Eckstein M, et al. Retinopathy of prematurity in middle-income countries. Lancet 1997;350:12-14.
25 Visser Kift E, Freeman N, Cook C, et al. Retinopathy of prematurity screening criteria and workload implications at Tygerberg Children's Hospital, South Africa: A cross-sectional study. S Afr Med J 2016;106:602-6.

26 Mutangana F, Muhizi C, Mudereva G, et al. Retinopathy of prematurity in Rwanda: a prospective multi-centre study following introduction of screening and treatment services. Eye 2020;34:847-56.

27 Asferaw M, Gilbert C, Kerie A. Retinopathy of prematurity - an emerging cause of childhood blindness in Ethiopia. Ethiop Med J 2020;58 https://emjema.org/index.php/EMJ/article/view/1377

28 Baiyeroju-Agbeja AM, Omokhodion SL. Screening for retinopathy of prematurity in Ibadan. Niger J Ophthalmol 1998;6:23-5.

29 Hariharan L, Gilbert CE, Quinn GE, et al. Reducing blindness from retinopathy of prematurity (ROP) in Argentina through collaboration, advocacy and policy implementation. Health Policy Plan 2018;33:654-65.

30 Silva JC, Zin A, Gilbert C. Retinopathy of prematurity prevention, screening and treatment programmes: progress in South America. Semin Perinatol 2019;43:348-51.

31 Keraan Q, Tinley C, Horn A, et al. Retinopathy of prematurity in a cohort of neonates at Groote Schuur Hospital, Cape town, South Africa. S Afr Med J 2017;107:64-9.

32 Jacoby MR, Du Toit L. Screening for retinopathy of prematurity in a provincial hospital in Port Elizabeth, South Africa. S Afr Med J 2016;106:598-601.

33 Mintz-Hittner HA, Kennedy KA, Chuang AZ, et al. Efficacy of intravitreal bevacizumab for stage $3+$ retinopathy of prematurity. $N$ Engl J Med 2011;364:603-15.

34 Wallace DK, Kraker RT, Freedman SF, et al. Assessment of lower doses of Intravitreous bevacizumab for retinopathy of prematurity: a phase 1 dosing study. JAMA Ophthalmol 2017;135:654-6.

35 Hartnett ME. Role of cytokines and treatment algorithms in retinopathy of prematurity. Curr Opin Ophthalmol 2017;28:282-8.

36 Seery CW, Betesh S, Guo S, et al. Update on the use of anti-VEGF drugs in the treatment of retinopathy of prematurity. J Pediatr Ophthalmol Strabismus 2020;57:351-62.

37 Wallace DK, Kraker RT, Freedman SF, et al. Short-Term outcomes after very low-dose Intravitreous bevacizumab for retinopathy of prematurity. JAMA Ophthalmol 2020;138:698-701.

38 Vinekar A, Gilbert C, Dogra M, et al. The KIDROP model of combining strategies for providing retinopathy of prematurity screening in underserved areas in India using wide-field imaging, tele-medicine, non-physician graders and smart phone reporting. Indian J Ophthalmol 2014;62:41-9.

39 Scruggs BA, Chan RVP, Kalpathy-Cramer J, et al. Artificial intelligence in retinopathy of prematurity diagnosis. Trans/ Vis Sci Technol 2020;9:5.

40 Enweronu-Laryea C, Dickson KE, Moxon SG, et al. Basic newborn care and neonatal resuscitation: a multi-country analysis of health system bottlenecks and potential solutions. BMC Pregnancy Childbirth 2015;15 Suppl 2:S4. 